\title{
GESCHICHTE UND KULTURGESCHICHTE
}

\section{VON WALTER GOETZ}

Niemand wird in Deutschland große Neigung tragen, Auseinandersetzungen $z u$ erneuern, die vor einem Jahrzehnt als sog. geschichtswissenschaftlicher Streit die Köpfe eine Weile erhitzten. Es erwies sich damals sehr deutlich, daß der Sinn der deutschen Historiker nicht nach langen philosophischen Diskussionen steht. Das hat Bernheim schon in den 8oer Jahren bedauert - er selber hat inzwischen durch den Ausbau des „Lehrbuchs der historischen Methode und (wie in der neuesten Auflage hinzugesetzt ist) der Geschichtsphilosophie" viel getan, die jüngere Generation mit diesen Fragen in Verbindung zu bringen. Jener geschichtswissenschaftliche Streit hat aber doch einige bestimmte Ergebnisse gehabt - in der weiteren Entwicklung wenigstens der historischen Arbeit in Deutschland und auch in Frankreich scheinen sie mir deutlich erkennbar zu sein. Die erdrückende Mehrzahl der kompetenten Forscher hat abgelehnt, was dem einen Teile der damals Streitenden als das Wesentliche erschien: die biologische Gesetzmäßigkeit der Geschichte, die Formulierung bestimmter Kulturzeitalter, die Vorherrschaft der sozialpsychischen Faktoren, die vergleichende Methode - das alles sind Anschauungen, die in Deutschland unterlegen sind und die auch durch die immer wiederholte Versicherung auf künftigen Sieg nichts an innerer Gewißheit gewinnen. $D a ß$ es in Deutschland nicht die beschränkte zünftige Gelehrsamkeit ist, die diese Theorien verständnislos ablehnt, zeigt wohl einwandfrei ihr Zurückweichen in Frankreich, wo der Boden für philosophische Bezwingung der Geschichte ein so viel günstigerer ist und wo dennoch das Hauptorgan solcher Anschauungen, die Revue de Synthèse historique, über allen naturwissenschaftlichen Schematismus weit hinausgewachsen ist.

Aber was viel stärker noch gegen eine Voreingenommenheit der deutschen Historiker in diesen Fragen spricht, ist die Tatsache, daß die jüngere Generation in Deutschland zu einem guten Teile dennoch zu einer Erweiterung des historischen Arbeitsfeldes, 
zu dem, was Lamprecht als Kulturgeschichte bezeichnet hat, hi neigt; je entschiedener diese Kreise Lamprechts geschich philosophisches und methodologisches System ablehnen, um ruhiger können sie bekennen, was sie den Anregungen Lamprecl zu verdanken haben. Freilich hat wohl der eine oder der ande: wie Lamprecht selber, diese Anregungen direkt von W. H. Rie von Gustav Freytag, von Jacob Burckhardt oder auch v Viktor Hehn erhalten - Janssens Geschichte des deutsch Volkes bedeutet in unvollkommener, v. Bezolds Geschichte c deutschen Reformation in vollkommener Weise die kulturgeschicl liche Bewältigung eines Zeitalters; Riezler hat seine Geschicl Baierns von Anfang an auf diese breitere Grundlage gestel und eine ganze Reihe von Werken der 70er und 8oer Jahre zeig die langsame Erweiterung des Rahmens der Darstellung. At wie dem auch sei - Lamprecht wurde der laute Prophet dies Gedanken, und da er sie (was immer mehr wert ist als die the retische Forderung) auf eine große Darstellung der gesamt deutschen Geschichte übertrug, so ist er schließlich doch viel stärl der Ausgangspunkt dieser Anregungen geworden als seine stre abgewandten Vorgänger. Daß er dann Eigenes, was sich mit dt Wollen der französischen Positivistẹn berührt, in starkem U: fang hinzugefügt hat, bedarf keiner weiteren Feststellung. I diesem Eigenen ist er zum größten Teile an dem Widerspruch c Fachgenossen gescheitert - aber auch das ist für diejenigen un1 uns, die wir noch Lernende waren, eine fruchtbare Anregung $\mathbf{z}$ Auseinandersetzung mit der eigenen werdenden geschichtlich Anschauung gewesen. Das möchte ich als das zweite, wenn au nur indirekte Ergebnis des geschichtswissenschaftlichen Streit bezeichnen: neben der Ablehnung der im engeren Sinne Lamprecl schen Theorien dennoch eine starke Zunahme des kulturgeschicl lichen Interesses bei der jüngeren Generation. Es liegt mir fer mich in theoretische Auseinandersetzungen einzumischen, für die i mich gar nicht genugsam zuständig fühle; aber die an einem neu Abschnitt des „Archivs für Kulturgeschichte" Beteiligten hab wohl das Recht, von der nun einmal gegebenen Sachlage zu sprech und die Aufgaben des Unternehmens zu prüfen. Anders freili 
uber diese Dinge nicht reden. Mir ist wohl bewußt, daß der Herausgeber dieses ",Archivs", der bekanntlich ein von Lamprecht völlig unabhängiger Kulturhistoriker ist und seinerseits an die Traditionen der Freytag, Burckhardt, Riehl anknüpfte, zwar nicht bezüglich der Stellungnahme gegen Lamprecht, wohl aber in anderen Punkten von mir verschieden denkt, wie er denn z. B. eine Scheidung $z$ wischen politischer und Kulturgeschichte, ferner die Einbeziehung des Volks - und gesellschaftlichen Lebens in das Arbeitsgebiet der Kulturgeschichte, endlich auch die besondere Wichtigkeit der sozialpsychischen Faktoren vertritt und die Bedeutung des Staates geringer einschätzt.

Ein negatives Ziel hätte das "Archiv" insofern, als es sich um die endgültige Beseitigung des Kulturgeschichtlichen im älteren Sinne handeln müßte. Seitdem es eine Volkskunde gibt, ist sie als die glückliche Erbin alles dessen anzusehen, was man früher so gern als Kulturgeschichte bezeichnete. „Kulturgeschichtliche Kuriosa", wie ein neuerer Autor ein Buch genannt hat, deuten mit dem Titel an, daß der Verfasser sich über den heutigen Begriff der Kulturgeschichte noch nicht ganz klar geworden ist. Was ins Gebiet der Volkskunde oder des gesellschaftlichen und Sittenlebens gehört, sollte nicht mehr als Kulturgeschichte bezeichnet werden. Freilich ist der Begriff Kulturgeschichte auch in wissenschaftlichen Kreisen noch strittig; Bernheim lehnt eine Gleichsetzung mit Universalgeschichte als zu vage ab und möchte nur ,die Geschichte der Entwicklung der sozialen Lebensformen und -prozesse, Arbeitsmittel und -resultate, geistigen wie materiellen, die vorwiegend aus der nichtpolitischen Tätigkeit der Menschen hervorgehen", damit bezeichnen. Er stellt neben diese Kulturgeschichte die politische Geschichte - diese beiden Wissenschaftszweige bilden dann also miteinander die Gesamtgeschichte. ${ }^{1}$ ) Aber es erhebt sich bei dieser Einteilung doch die Frage, ob das politische Leben nicht auch Kulturleben ist, ob der Staat als scheinbar außerhalb der Kultur stehend bezeichnet werden darf.

') Ähnlich wuinscbte Gothein, Die Aufgaben der Kulturgeschichte (1889), neben der politischen Geschichte eine selbständige Kulturgeschichte Dagegen schon Below, Gött. Gel. Anz. 1892, S. 286: er wüncht Vereinigung beider mit dem Nachdruck auf der politischen Geschichte. 
Die den Staat schlechtweg dem Kulturleben überordnen wollen, werden mit solcher Begriffsbestimmung einverstanden sein; die anderen aber - den Staat als Ergebnis des gesamten Kulturlebens ansehend - werden in einer solchen Scheidung von Kulturgeschichte und politischer Geschichte eine Zerreißung innerer Zusammenhänge erblicken - für sie wird Lamprechts Definition von Kulturgeschichte als Zusammenfassung alles geschichtlichen Geschehens unter dem Gesichtswinkel der Entwicklung (freilich ohne Gesetzmäßigkeit!) sympathischer sein. Vielleicht wäre es noch besser von Allgemeiner Geschichte oder von Gesamtgeschichte zu sprechen - aber Begriffe haben ihr eigenes Leben, und das Wort Kulturgeschichte ist heute bereits verständlicher als irgendein anderes Wort: wer eine Vorlesung über Kulturgeschichte des Mittelalters anzeigt, kann darauf rechnen, daß die Studenten darunter eine Zusammenfassung von politischer, wirtschaftlicher, geistiger Geschichte des Mittelalters verstehen. Am besten wäre es allerdings, wenn mit dem Worte Geschichte so selbstverständlich eine Gesamtgeschichte verstanden würde, daß es der andern Begriffe nicht bedürfte - dann müßten freilich alle Spezialwerke sich als solche kennzeichnen: als politische Geschichte des Mittelalters usw. Wenn heute hie und da Kulturgeschichte mit Geistesgeschichte gleichgesetzt wird, so schleicht sich dabei ein Irrtum ein: steht auch gewiß hinter allem geschichtlichen Geschehen der denkende und handelnde Mensch, so ist die Geschichte des geistigen Lebens doch nur ein Bestandteil der Gesamtgeschichte, über dessen Wichtigkeit man streiten kann wie über die Wichtigkeit des Staates für das geschichtliche Leben (s. unten !). Der Begriff Kulturgeschichte bleibt ein Hilfsbegriff wie jeder andere; aber er deckt sich mit der zu bezeichnenden Sache besser als irgendein anderer.

Die wichtigere Frage ist, ob der Historiker es als seines Amtes ansehen darf, Geschichte in diesem unendlichen Umfang zu treiben. $\mathrm{DaB}$ die politische Geschichte nicht die gesamte Geschichte ist, bestreiten auch ihre einseitigsten Vertreter nicht; wie hoch man auch die Geschichte des Staates einschätzen will - es bleibt daneben ein großes Leben übrig, das ebenfalls Geschichte ist. Was die politischen Historiker behaupten, ist ja nur, daß der Staat 
das Wesentliche im geschichtlichen Verlaufe sei und daß das Arbeitsgebiet des Historikers, der Begrenztheit der menschlichen Kräfte entsprechend, auf diesen wesentlichen Teil der Geschichte zu beschränken sei. Die Forderung der Beschränkung soll die Grundlage wissenschaftlicher Vertiefung sein. Dabei bleibt die Frage offen, wer denn das Recht zur Zusammenfassung alles geschichtlichen Lebens habe - es sei denn, daß man grundsätzlich solche Zusammenfassung als unmöglich ablehne.

In der Zeit der Beschaffung, Ordnung und Durchprüfung eines ungeheuren Quellenstoffes, in der Zeit des Aufbaues einer gesicherten historischen Methode war die Beschränkung vieler Forscher auf die politische Geschichte begreiflich, ja notwendig. Aber wie oft ist schon damals die Grenze überschritten worden, wie viele gute und fruchtbare, ja glänzende Werke danken dem Drange nach weiterem Ausblick in das geschichtliche Leben ihre Entstehung. Neben den eigentlichen Kulturhistorikern hat Treitschke doch gewiß alle Fesseln rein politischer Geschichtschreibung gesprengt. Die besondere Aufgabe hat zudem jederzeit zur Abweichung von vorgeschriebenen Methoden geführt: Reformationsgeschichte hat tiefgründig niemals ohne starke theologische Anleihen, die Geschichte der Französischen Revolution nicht ohne philosophische Studien, die Geschichte jedes großen Krieges nicht ohne militärische Kenntnisse geschrieben werden können. Auch der politische Historiker steht, wenn er nicht hinfälliges Stückwerk geben will, uberall an den Grenzen des eigenen und vor den Toren fremden Wissens - er muß sie sich offnen, wenn er in das Wesen der Dinge eindringen will. Liegt nicht schon in der Forderung, daß die Geschichtsforschung den Staat, seine Verfassung und sein Recht schildern solle, der Zwang zur Überschreitung selbstgezogener Grenzen? Denn Verfassung und Recht sind Arbeitsgebiete des Juristen und nicht des Historikers. Wenn die deutsche Verfassungsgeschichte vorwiegend von Historikern ausgebaut worden ist, so zeigt dies nur aufs deutlichste, daß der Historiker je nach seinen besonderen Aufgaben das Recht zur Arbeit auf Nachbargebieten hat. So ist auch der Gang der Dinge gewesen: wollte man die geschichtlichen Meisterwerke des I9. Jahr- 
hunderts zusammenstellen, so würde sich ergeben, daß ihre Verfasser je nach Neigung und Veranlagung nach allen Richtungen des geschichtlichen Lebens hin gearbeitet haben, und daß die Geschichte der Geschichtschreibung gerade in ihren besten Leistungen eine bestimmte Begrenzung nicht kennt. Ranke selber hat sich, wenn auch zaghaft, auf das Gebiet der Geistesgeschichte begeben, als er seine Abhandlung ,Zur Geschichte der italienischen Poesie" schrieb (Werke Bd. 5I, S. I55ff.). Wenn die Forderung nach Schilderung alles geschichtlichen Lebens erst am Ende des 19. Jahrhunderts erhoben worden ist, so liegt der Grund dafür nicht in dem Widerspruch der historischen Methode, sondern allein am Mangel bestimmter Voraussetzungen: eine gewisse Aufarbeitung der engeren Wissenschaftsgebiete muß erfolgt sein, eine starke Literatur der Grenzuberschreitungen muß vorhanden sein, ehe der Gedanke an eine Verbindung aller geschichtlichen Lebensgebiete entstehen kann. An einem bestimmten Zeitpunkt wissenschaftlicher Arbeit werden vorher unmögliche Aufgaben möglich - das ist der Gang aller wissenschaftlichen Entwicklung.

Und es ist eine Notwendigkeıt, daß solche neue und größere Ziele aufgestellt werden. Auch die Wissenschaft kennt das Gesetz der Ermüdung an Idealen. Wenn dem Historiker das Arbeitsgebiet auf die Dauer in gleichmäßiger Begrenzung vorgeschrieben sein sollte, würde diese Wissenschaft schließlich zu einer dumpfen Reparaturwerkstätte herabsinken: kleine Erweiterungen des Gebietes, immer kleinlichere Verbesserungen der Vorgänger und im ganzen eine unerträgliche Summe von Wiederholungen würde das Ergebnis sein. Eine Wissenschaft, die an Gründlichkeit und an Langeweile, d. h. an Unfruchtbarkeit zugrunde gehen würde; eine Wissenschaft zudem, die eines der obersten Gesetze ihres Daseins vergessen hätte: sich mit dem Leben der gesamten Nation verbunden $z u$ fuhlen. Die Geschichtswissenschaft kann ihre große Stellung als Lehrmeisterin der Nation nur bewahren, wenn sie den Erweiterungen alles Lebens auch ihrerseits Rechnung trägt - das heißt im engeren: wenn sie die Ergebnisse einer auf allen Gebieten vertieften Einzelforschung immer wieder in große, verständliche Zusammenhänge zu setzen vermag. Wenn die 
Gelehrten das nicht zustande bringen, so geht die Nation zu den Dilettanten - womit sich die Aufgabe aller echten Wissenschaft von selber ergibt. Der Wille zu tieferen Einblicken in die Zusammenhànge des geschichtlichen Lebens wäre selbst dann noch berechtigt, wenn wir vor unlösbaren Aufgaben ständen. Denn die Grundlichkeit allein ist doch nur ein scheinbarer Gewinn einer Wissenschaft; ihr Leben beruht in gleicher Weise auf dem Vorwartsschreiten im Zusammenhang mit der Gesamtwissenschaft, mit den Erweiterungen des allgemeinen Denkens - selbst mit dem bloßen Suchen einer Zeit darf lebendige Wissenschaft Fuhlung halten.

Der Hinweis freilich auf die bei einer Gesamtgeschichte auch des kleinsten Zeitraumes notwendigen Übergriffe in völlig fremde Gebiete wie Naturwissenschaft und Technik kann den Glauben an die Möglichkeit so umfassender Geschichtsschreibung erschüttern. Die Aufgabe für unmöglich erklären bedeutet aber nichts anderes als ein Sichzurückziehen aus der geistigen Weiterarbeit der Nation; die Aufgabe nur gerade dem Historiker verweigern, weil sie seines Amtes nicht sei, wäre eine Bescheidenheit, die uns nur Mißachtung eintragen konnte. Auch vernichtet jedes Zuviel an Bescheidenheit das Selbstvertrauen, aus dem die starken Leistungen allein hervorgehen. Es fragt sich, ist die Erweiterung der Aufgaben der Geschichtswissenschaft wirklich so unmöglich?

Die Altertumswissenschaft hat niemals die Grenzen gekannt, die die Geschichtswissenschaft sich selber setzen möchte. Der klassischePhilologe hat jederzeit die Gesamtheit der antiken Kultur als sein Arbeitsgebiet angesehen, bis hin zur antiken Medizin, Naturwissenschaft oder Technik. Die Ägyptologen haben in der Schilderung der ägyptischen Gesamtkultur ihre Aufgabe gesehen, genau so wie die Theologen uns judische „Kulturgeschichten" geschrieben haben. Mommsen hat sich eine Darstellung der römischen Geschichte nicht denken können ohne Einbeziehung von Wirtschaftsleben und Religion, Literatur, Kunst und Wissenschaft. Eduard Meyers Geschichte des Altertums ist Kulturgeschichte in unserem Sinne. Man rennt im Grunde offene Türen ein, wenn man solche Geschichtsauffassung gegenüber einer rein 
politischen verteidigen will.1) Und niemand kann heute noch behaupten, es sei der geringere Umfang des Materials, der für das Altertum solche Zusammenfassung ermögliche - das Material ist auch in diesen Gebieten ins Uferlose angewachsen. Was aber für das Altertum möglich ist, darf auch für Mittelalter und Neuzeit möglich sein. Es war gewiß vor Jahrzehnten eine unlösbare Aufgabe, eine wissenschaftlich haltbare Kulturgeschichte des deutschen Mittelalters zu schreiben; wie haben sich aber seitdem die verschiedensten Wissenschaften gegenseitig in die Hände gearbeitet, wie viele Vorarbeiten erleichtern die Aufgabe! Vor allem für die historischen Vorlesungen sollte man als Ziel eine möglichst umfassende geschichtliche Darstellung festhalten. Den künftigen Lehrern ist mit der ausführlichen Wiedergabe jedes Romzugs und jeder politischen Verhandlung herzlich wenig gedient - die herkömmliche Breite in diesen Dingen sollte energisch beschränkt werden zugunsten eines Einblicks in das gesamte mittelalterliche Leben. Es ist den Studenten nicht möglich, über Literaturgeschichte und Kunst, Wirtschaftsleben und Kirchengeschichte, Philosophie und Wissenschaftsgeschichte Spezialvorlesungen zu hören; der Historiker muß die Aufgabe ubernehmen, einen Teil dieser Spezialvorlesungen zu ersetzen und das bei allen andern Getrennte wieder zur geschichtlichen Einheit zusammenzufügen. Nur so ist für den künftigen Lehrer eine historische Anschauung erreichbar.

Daß es sich dabei nicht um eine Ersetzung der Spezialvorlesungen im vollen Sinne handeln kann, ist klar. Dem Kunsthistoriker oder dem Philosophen usw. sich gleichzusetzen, wäre eine lächerliche Anmaßung des Kulturhistorikers. Er lebt von ihrer Arbeit, aber er trägt in alle Anleihen ein Neues, sein Eigenes hinein, indem er die gegenseitige Abhängigkeit der einzelnen Lebensgebiete aufdeckt, ihre jeweilige Bedeutung für das Gesamtleben und für die geschichtliche Weiterentwicklung feststellt und den einheitlichen Kräften, die dieses vielgestaltige Leben treiben,

3) In der soeben erschienenen "Einfuihrung in das Studium der neueren Geschichte" lehnt Gustav Wolf die Möglichkeit einer Gesamtgeschichte für die neuere Zeit ab und empfiehlt Beschränkung auf die politische Geschichte (S. 19). Hie und da sind also die Türen doch noch geschlossen! 
nachspürt. Damit ergeben sich sofort dieGrenzen kulturhistorischer Forschung und Darstellung: nur das für die geschichtliche Weiterentwicklung Wesentliche soll aus den Arbeitsgebieten anderer Wissenschaften herausgeholt werden. Niemals soll also die Geschichte der Naturwissenschaften Aufgabe kulturhistorischer Darstellung sein, sondern allein die Bedeutung naturwissenschaftlicher Fortschritte für das geschichtliche Leben soll geschildert werden: zur Klärung mancher sonst unverständlichen Erscheinungen dieses geschichtlichen Lebens. Wenn ich statt geschichtlichen Lebens sagen wollte: des staatlichen Lebens, so würde sich das eben Formulierte nicht von demjenigen unterscheiden, was weitsichtige politische Historiker als Aufgabe unserer Wissenschaft bezeichnet haben. Und ich stehe auch gar nicht an, den Staat als den wesentlichen Mittelpunkt dieses geschichtlichen Lebens zu bezeichnen. Er ist fur einen großen Teil des Kulturlebens das ordnende, das bedingende Prinzip. Aber wenn sich "in der Wechselwirkung zwischen Gesellschaft und Staat" die Geschichte vollzieht, ${ }^{1}$ ) so ist a aßßeren Einwirkungen auf den Staat ein geschichtlicher Platz eingeräumt, und diesen Einwirkungen nachzugehen ist genau so gut die Aufgabe des Historikers wie die Geschichte des Staates im engeren. Der Staat führt unter allen Kulturmächten das stärkste Dasein, alles andere stärker bestimmend als er selber bestimmt wird - aber seine Entwicklungen sind nicht seine eigene Arbeit allein, sondern hinter ihm stehen Mächte, die ihn zeitweise beherrschen oder in neue Bahnen hineinzwingen: religiöse, wirtschaftliche, geistige Mächte. Auch der Staat steht unter dem Gesetz der Wechselwirkung aller geschichtlichen Kräfte, und so ist er nur ein Teil des Ganzen. Weil er aber die umfassendste geschichtliche Macht ist, wird auch die Kulturgeschichte im Sinne einer Gesamtgeschichte den Staat grundsätzlich nach Móglichkeit in den Mittelpunkt der Arbeit zu stellen haben, mit dem Vorbehalt freilich, daß auch der Staat ein bedingtes geschichtliches Leben führt ${ }^{2}$ ). Daß in letzter

1) Moriz Ritter, Der Streit zwischen politischer und Kulturgeschichte. Beilage zur Allg. Ztg. 1893, Nr. 262.

2) Die Bedeutung des Staates im Verhältnis zur Kultur ist von Schafer, Geschichte und Kulturgeschichte (I89I), von Below (Gött. Gel. Anz. I892, 
Linie die Kulturgeschichte eine Geistesgeschichte sei, weil hinter Staat und Kirche, Wirtschaft und Kunst usw. der denkende Mensch stehe, ist eine nicht völlig einleuchtende Hypothese. Die aktiven Seelenkräfte des Menschen vermögen die Entwicklung des Staates zu bestimmen; aber der Staat vermag diese Seelenkräfte in lange, jahrhundertelange Passivität zu zwingen, und wenn man dem Staat diese Macht nicht zuerkennen wollte, müßte man physiologische Voraussetzungen des Seelenlebens als eine Kraft anerkennen, die auf die seelischen Entwicklungsstufen treibend und hemmend einwirkt. Der geschichtliche Stillstand eines Volkes oder irgendeine geschichtliche Entwicklung wie z. B. die religiöse des Abendlandes und des Orients geht in letzter Linie vielleicht stärker auf physiologische als auf psychische Bedingungen zurück. Aber wie schwankend ist dieser Boden geschichtlicher Verursachung! Vielleicht sind kunftig einmal tiefere Einsichten möglich; heute kann man doch nicht anders, als auf solchen Gebieten höchste Zurúckhaltung üben. Man kommt zunächst wohl kaum über das Gesetz der Wechselwirkung der geschichtlichen Kräfte hinaus - womit der Geistesgeschichte ihr volles Recht neben den anderen Gebieten gewahrt bleibt, womit aber auch dem Staate eine Vormachtstellung im geschichtlichen Leben eingeräumt werden darf.

Das Ergebnis also wäre: die letzte Aufgabe der Geschichtswissenschaft ist eine Gesamtgeschichte der Entwicklung des Menschengeschlechts vorwiegend innerhalb seiner staatlichen Verbände (= Kulturgeschichte). Theoretisch entfernt sich diese Anschauung nicht allzuweit von der Meinung der politischen Historiker, die in eine Staatengeschichte alles geschichtliche Leben einfügen wollen. Auf das Gleichmaß der Ausführung kommt es freilich an: 500 Seiten politische Geschichte und 50

S. 287 ff.) und von Ritter (a. a. O.) so ausführlich erörtert worden, daß eine neue Diskussion kaum weiter führen würde. Über die Bedeutung des Staates mit den vorgenannten völlig einig, kann ich mir den Staat doch nicht anders als innerhalb des Kulturlebens denken, von ihm bestimmt und es selber wieder bestimmend. Die Religion des alten Ägyptens ist zeitweise wichtiger als der Staat; ebenso steht es mit der Kultur der Renaissance. Und beim Mittelalter könnte man schwanken, ob nicht Religion und Kirche wichtiger sind als der Staat. 
Seiten „Kultur" ist weder eine Verarbeitung noch ein ausreichendes Abwägen der miteinander wirkenden geschichtlichen Kräfte. Johann Friedrich Böhmer wird kaum als kulturgeschichtlicher Phantast angesehen werden, und doch stammt von ihm der Satz: „Als eigentliches Objekt einer Geschichte denke ich mir die Volkspersönlichkeit, ihre Urzustände, ihre innere Entwicklung, ihre äußeren Verhältnisse, ihr Absterben, also ihr Lebensalter, ihre Jahreszeiten; die politische Geschichte der Kern ..." "1) In durchaus schlechter Gesellschaft befindet sich also der Kulturhistoriker der Gegenwart nicht. Was ihn diskreditiert hat, ist das Übermaß des Neuen gewesen, das er - nach einigen - bringen sollte: an Stelle einer naturgemäßen Erweiterung seines geschichtlichen Arbeitsfeldes auf den Grundmauern bisher geleisteter Arbeit vermaß man sich, mit einer naturwissenschaftlich aufgebauten Kulturgeschichte die Geschichte überhaupt erst zum Range einer Wissenschaft $\mathrm{zu}$ erheben. Man kann dem Enthusiasmus viel zugute halten - die großen Fortschritte der Wissenschaft wurden aber zumeist ohne Zuhilfenahme lauter Reklame gemacht. Auch auf die Erfindung einer neuen Methode hat man sich viel zugute getan - aber diese ,vergleichende" Methode der historischkritischen gleichsetzen und die neue Wissenschaft der Geschichte darauf aufbauen, heißt doch das Exakte mit dem Inexakten stuitzen. Wenn der neuen Schule heute mit Recht Verwilderung der wissenschaftlichen Arbeit vorgeworfen wird, so verdankt sie dies dem schwankenden Boden, auf den sie sich selber gestellt hat. $\left.{ }^{2}\right)$

1) J. Janssen, Böhmers Leben S. 19I. - In einem Brief an Janssen von 1854 hält Böhmer die Forderung einer mehr kulturhistorischen Richtung für wohl begründet, aber er spricht sich für eine Trennung von Kulturgeschichte und politischer Geschichte aus (Böhmers Leben und Briefe III S 1 18; vgl. auch I S. 357). Man sieht daraus, wie Janssen zu seiner kulturgeschichtlichen Darstellung gekommen ist.

2) Das Urteil Ernst Troeltschs über die wissenschaftlichen Qualitäten der Arbeiten aus Lamprechts Schule hat eine gereizte Erwiderung Lamprechts im Lit.Zentralblatt 1909 , n. 45 (6. Nov) hervorgerufen. Und doch ist, was Troeltsch ausgesprochen hat, so ziemlich das allgemeine Urteil uber einen großen Teil der Leistungen der Lamprechtschen Schule. Wenn man uber das literarische Porträt des Io. Jahrhunderts auf Grund allein der Übersetzungen in den "Geschichtsschreibern der deutschen Vorzeit" 
Jede Erweiterung der wissenschaftlichen Arbeit wird auch Erweiterungen der Methode hervorrufen. Da es sich bei der Zusammenfassung spezialwissenschaftlicher Forschungen $\mathrm{zu}$ einer Gesamtgeschichte nicht um eine bloße Kompilation handelt (wie Gustav Wolf in seiner ,Einführung in das Studium der neueren Geschichte" S. I9 irrigerweise annimmt), sondern um die Ergrundung des Gemeinsamen und Entgegenstrebenden, der Einwirkung des einen Kulturgebiets auf die andern, der Bedeutung jedes einzelnen Gebietes für die Weiterentwicklung des geschichtlichen Gesamtlebens handelt, so bedarf es in der Tat neuer methodischer Maßstäbe. Die meisten dieser Fragen liegen auf dem Gebiete der Geistesgeschichte, so daß diese zu einem der wichtigsten Hilfsmittel der Kulturgeschichte wird. So fällt die Ausbildung einer geistesgeschichtlichen Methode als eine der nächsten und nötigsten Aufgaben dem Kulturhistoriker zu. Auch der kleinste ernsthafte Versuch auf diesem Gebiete wird aber zeigen, daß eine solche geistesgeschichtliche Methode nur unter Voraussetzung der historisch-kritischen bestehen kann. Man suche doch z. B. die geistige Stellung eines

schreibt wie Kircheisen, wenn man das literarische Porträt bei Macchiavelli schildert lediglich auf Grund der "Florentiner Geschichten" und ohne Zugrundelegung der italienischen Texte wie Kemmerich, wenn man „das älteste japanische Seelenleben nach seinen literarischen Ausdrucksformen" ohne sicher datierte Literaturwerke und ohne Kenntnis des Japanischen bestimmt wie Leo, wenn man das chinesische Seelenleben auf Grund von Holzschnitten zeichnet, hinsichtlich deren Datierung die Gelehrten um Jahrhunderte voneinander abweichen wie Hoerschelmann, wenn man die Barotse schildert ohne genügende ethnographische Vorkenntnisse wie Martin Richter - und wenn hinter dem allen das ewig gleiche Schema gesetzmäßiger kuiturgeschichtlicher Entwicklung steht, so darf sich das Haupt der Schule uber zweierlei nicht wundern: daß man diese Arbeiten nicht als wissenschaftlıch betrachtet und $\mathrm{da} \beta$ man die fortwährende Versicherung, in dieser Schule werde nicht auf die Worte des Meisters geschworen, als ein Zeichen bösen Gewissens ansieht. Fügt man noch hinzu, daß Schaumkells Schrift uber die deutsche Kulturgeschichtschreibung als ein Plagiat bezeichnet worden ist, so geniigt wohl diese kleine Übersicht zur Bestätigung von Troeltschs Urtell! Einzelne dieser Themen - andere sind in einem von Lamprecht herausgegebenen Reklameheft über die Arbeiten seiner Schuler enthalten - uberschreiten die Grenzen der Geschichtswissenschaft durchaus und sind Eingriffe in fremde Gebjete, bei denen die Bearbeiter notwendig scheitern müssen. 
Geschichtschreibers und seines Werkes zu bestimmen ohne fortgesetzte kritische Prüfung des Eigenen und des Entlehnten, des Historisch-Zuverlässigen und des Erdichteten, der genauen Entstehungszeit seines Werkes - überall beruht die geistesgeschichtliche Forschung in ersterLinie auf der historisch-kritischen, ehe sie ihre eigenen Maßstäbe anlegen kann. Die Annahme von historischen Gesetzen, der Glaube an die Exaktheit einer vergleichenden Methode verdrängt mit Notwendigkeit in der Geschichtswissenschaft jede induktive Forschung - der Beweis ist schon reichlich genug geliefert, daß diejenigen, die die Geschichte zum Rang einer exakten Wissenschaft erheben wollten, der Deduktion die Tore weit geöffnet haben. ${ }^{1}$ ) Dabei soll gewiß nicht Untersuchungen uber Analogien, uber das scheinbar Gesetzmäßige gewisser geschichtlicher Erscheinungen, über die letzten treibenden Faktoren des geschichtlichen Lebens, über die Bedeutung von Individuum und Gesamtheit im geschichtlichen Verlaufe usw. der Boden entzogen werden: die deutsche Geschichtswissenschaft bedarf unzweifelhaft einer fortgesetzten Erörterung dieser Fragen im Anschluß an alle neueren geschichtsphilosophischen, soziologischen, ethnographischen und ähnliche Forschungen; aber es ist ein anderes, diese Wissensgebiete fur höchst beachtenswert ansehen oder halbfertige Wissenschaften zur Grundlage historischer Forschung machen wollen. Die Kulturgeschichte als Wissenschaft hat zunächst nur Aussicht auf Anerkennung, wenn sie aus dem Zustand eines vagen Enthusiasmus auf den Boden methodischer Arbeit gefuhrt wird. Wie kann man Gesetze für

') Man vergleiche Hoerschelmanns reizenden Einfall in seiner Arbeit uber "die Entwicklung der altchinesischen Ornamentik". S. 3 stellt er fest, daß jede Möglichkeit einer absoluten Chronologie bei den altchinesischen Kunstwerken fehlt. Er glaubt deshalb einen anderen Weg einschlagen zu müssen. „Das wiederholte eingehende Studium der Abbildungen befestigte in mir mehr und mehr die Überzeugung, da $\beta$ in diesen verschiedenartigen Gebilden nicht das regellos wirre Durcheinander von Ausgeburten einer bizarren Phantasie vorliege, sondern daß es sich um Kunstwerke handle, welche von Menschen durchaus verschiedener Kulturstufen in gesetzmäßiger Aufeinanderfolge im Laufe von Jahrtausenden geschaffen worden seien." Man sieht, daß es für diese Art von Kulturgeschichte keinerlei kritische Schwierigkeiten mehr gibt! Man konstruiert sich die Dinge so, wie man sie haben möchte. 
die Entwicklung des geistigen Lebens einer Nation aufstellen, wenn das geistige Leben ganzer Perioden noch so gut wie unerforscht ist und wenn vor allem die Grundsätze seiner Ermittlung noch fehlen? Mit Eindrücken und subjektiven Wahrnehmungen sst da nichts getan; es handelt sich vielmehr um ein Sammeln und Abwägen, um ein Eindringen in Motive und um eine Schärfung der Beobachtung, wie sie uns z. B. dem Mittelalter gegenüber heute noch zum guten Teil fremd ist. Aber es ist hier nicht die Stelle, die Grundsätze einer geistesgeschichtlichen Methode zu entwickeln; es kommt nur darauf an, mitten aus der Arbeit über solche Fragen heraus festzustellen, daß die historisch-kritische Methode die Voraussetzung, der Unterbau jeder geistesgeschicht. lichen Arbeit ist - wie die Mathematik die Voraussetzung jeder Technik. Damit ergeben sich Verbindungen zur Rankeschen Schule - so sei etwas einseitig die bedeutendste Richtung in der deutschen Geschichtswissenschaft des 19. Jahrhunderts genannt die jeden inneren Gegensatz aufheben und jede Großmannssucht der kulturgeschichtlichen Richtung ausschließen. Das Neue ist die notwendige Entwicklung unzähliger Keime dieser älteren Geschichtswissenschaft, ist die Fortführung der wissenschaftlichen Arbeit unter dem Einfluß vorwärtsstrebender Nachbarwissenschaften und unter der Einwirkung der allgemeinen Erweiterung unseres Denkens.

Dem Ideal steht die praktische Durchfuhrung auch hier gegenuber. Lehrbar und lernbar ist die vollkommene Geschichtsschreibung so wenig wie das künstlerische Meisterwerk. Man kann immer nur gewisse Vorbedingungen lehren und lernen. Jede neue wissenschaftliche Richtung ist in der üblen Lage, dem eingeschulten Personal einer herrschenden Richtung nur ungeschulte Kräfte gegenüberstellen zu können. So ist auch die Kulturgeschichte bei dem Mangel methodischer Richtpunkte gewiß noch im Rückstand gegenüber innerlich so gesicherten Gebieten, wie es z. B. die politische Geschichte, die Verfassungsund Wirtschaftsgeschichte sind. Aber da eine jede Wissenschaft solche Zeit des Werdens durchgemaght hat, so wird man schwerlich daraus einen Vorwurf ableiten können - es sei denn, daß einer solchen Richtung der ernste Wille $z \mathrm{u}$ wissenschaftlicher 
Vertiefung fehle. Wie alle junge Wissenschaft wird aber die Kulturgeschichte die Pflicht haben, bei der Verkündigung ihrer Resultate Zurückhaltung $z u$ üben und die lernende Generation $z u$ wissenschaftlicher Gewissenhaftigkeit zu erziehen. Der historische Unterricht unserer Universitäten darf nicht auf Kulturgeschichte zugeschnitten werden. Die Zukunft unserer Wissenschaft beruht nicht auf dem Überwiegen von Kulturhistorikern, sondern auf dem Vorhandensein von Historikern aller Art. Das klassische Geschichtswerk kann sein, was es will - ein Gesetz dafür gibt es nicht. Und das Blühen der Wissenschaft beruht auf dem Bluhen aller einzelnen Zweige. Nur dann wird hie und da dem einzelnen die große Zusammenfassung gelingen.

Aber nicht nur, weil die Züchtung von Kulturhistorikern unerwünscht wäre, soll die Kulturgeschichte auf der Universität vor Übermaß bewahrt bleiben, sondern auch weil die Erziehung zum Historiker allein durch die historischen Unterdisziplinen und nur mit Hilfe der historisch-kritischen Methode geschehen kann. Nur wer diese gelernt hat, sollte in das kulturgeschichtliche Arbeitsgebiet eingefüht werden. Und auch da wird der Lehrer die Aufgaben so zu wählen haben, daß der Schüler vom festen Boden aus zu sammeln und zu beobachten hat. Durch Doktoranden das Seelenleben von Jahrhunderten darstellen zu lassen, gehört in jedem Falle in ein Gebiet, wo die wissenschaftliche Diskussion aufhört.

Was die Kulturgeschichte aber bei solcher im eigensten Interesse liegenden Beschränkung zu fordern hat, ist nicht nur die Anerkennung ihres Daseins und ihrer Ziele, sondern auch die Verstattung eines bestimmten Raumes innerhalb des Universitätsunterrichts. Die großen Vorlesungen über deutsches Mittelalter und deutsche Neuzeit (beim Altertum ist es zumeist selbstverständlich) müssen streben, allgemeine Geschichtsdarstellung $z \mathrm{u}$ sein. So gut wie Vorlesungen über Weltgeschichte ein Bedürfnis sind (selbst wenn sie rein politisch wären, damit doch wenigstens von einer Seite her das Leben aller Völker in den Gesichtskreis des Lernenden träte), so gut muß auch die deutsche Geschichte dem künftigen Lehrer in ihrem vollen Umfang erschlossen werden. Mag selbst das Schreckliche geschehen, daß 
der Lehrer dabei Irrtümer begeht (die übrigens auch der politische Historiker regelmäßig begeht, sei es nun durch Unkenntnis des 2o. Aufsatzes über denselben Gegenstand oder durch subjektive Deutung der Tatsachen), so bleibt das Gesamtbild doch wesentlicher als der Teil, der für das Ganze gegeben wird. Es bleibt Raum genug, in Spezialvorlesungen die Einzelfàcher mit voller Grundlichkeit zu behandeln. Vor allem aber werden diehistorischen Übungen ganz uberwiegend der historisch-kritischen Methode in ihrem weitesten Umfang vorzubehalten sein - an diesem Punkte darf die Kulturgeschichte nicht mehr fordern, als den gereifteren Semestern ein neues Gebiet zu erschließen. Denn nur wer Gründlichkeit und Gewissenhaftigkeit von der historischkritischen Methode gelernt hat, wird für weitergehende Arbeiten fähig sein.

Was hiermit fur die Kulturgeschichte gefordert wird, ist sicher kein Radikalismus. Auch nichts Neues, im Grunde etwas Selbstverständliches. Es kam mir darauf an, eine vorhandene Lage zu schildern; wenn ich nicht überall das Richtige getroffen haben sollte, so darf ich dennoch behaupten, daß ein großer Teil der jungeren Generation von ahnlichen Gedanken beherrscht ist. Und das ist eine Tatsache, die für die Weiterentwicklung unserer Wissenschaft bedeutsam ist - die heranwachsende Historikergeneration der sechziger und siebziger Jahre des 19. Jahrhunderts hat zum großeren Teile noch anders gedacht und sah in anderen Arbeiten ihr Ideal. Strebt heute die jüngere Generation anderen Zielen zu, so hat sie ein gutes Recht dazu, denn nur die selbsterlebten Ideale sind unser Eigentum und Förderer unserer Schaffenskraft. Aber die vorstehenden Ausführungen wollten zeigen, wie Neues und Altes sich verbinden läßt und sich verbinden muß, wenn eine Verwilderung unserer Wissenschaft aufgehalten werden soll. 